\title{
Helicobacter pylori does not release cysteamine into gastric juice
}

\author{
M B Leonard, W D Neithercut, D Gillen, K E L McColl
}

\begin{abstract}
Aim-To determine whether Helicobacter pylori releases cysteamine into gastric juice as cysteamine is known to be ulcerogenic.

Methods-Samples of fasting gastric juice were collected from 22 individuals (four women); 10 subjects were $H$ pylori negative. The presence of infection was confirmed by examination and culture of gastric biopsies. Cysteamine in gastric juice was measured by reversed phase gradient high performance liquid chromatography with a detection limit of $10 \mu \mathrm{mol} / 1$.

Results-Cysteamine was not detected in any of the gastric juice samples or in extracts of cultured $\mathrm{H}$ pylori.

Conclusions-If $H$ pylori produces cysteamine then the amounts produced are insignificant and are unlikely to explain the association between $H$ pylori infection and the development of duodenal ulcer disease.

(F Clin Pathol 1997;50:769-770)
\end{abstract}

Keywords: Helicobacter pylori; cysteamine; duodenal ulcer

Helicobacter pylori, which colonises the gastric mucosa and is thought to be the cause of the development of duodenal ulcer disease, is a metabolically unusual microorganism.

It has been recognised since the organism was first identified that it possesses marked amounts of urease activity. ${ }^{1}$ It appears to use this enzyme to scavenge nitrogen from urea that diffuses into the gastric juice from the blood stream. ${ }^{2}$ Nitrogen from urea is assimilated into amino acids through the action of the glutamate dehydrogenase and glutamine synthetase enzymes, both of which are expressed by $H$ pylori.

During studies that followed the incorporation of ${ }^{15} \mathrm{~N}$ labelled nitrogen from urea into amino acids it was noticed that a significant amount of ${ }^{15} \mathrm{~N}$ labelled nitrogen was incorporated into cysteine. This amino acid is structurally related to cysteamine, which is a moiety incorporated into pantotheine through sulphur containing amino acid metabolism. ${ }^{4-6}$

Cysteamine itself has been used in rats to induce experimental duodenal ulcer disease. ${ }^{78}$ The amine is thought to induce ulcers by depleting the concentration of somatostatin in the gastric mucosa thereby impairing the inhibitory control of gastric acid secretion. The mechanism by which $H$ pylori predisposes to ulcer disease is also via depletion of mucosal somatostatin ${ }^{10}$ and disruption of the somatostatin mediated inhibitory control of acid secretion. ${ }^{11-13}$

The observation that sulphur containing amino acids were manufactured in response to urease activity suggested the possibility that cysteamine production and release by $H$ pylor $i$ might be the mechanism by which infection of the gastric mucosa by this microorganism causes duodenal ulcer disease as well as type B gastritis.

\section{Patients and methods}

PATIENTS

Samples of fasting gastric juice were collected from 22 individuals (four women) following passage of a 16 French nasogastric tube (Anderson Inc, New York, USA). Of 22 individuals studied 10 (two women) were $H$ pylori negative volunteers, seven (one woman) had $H$ pylori infection and duodenal ulcer disease, and five (one woman) had $H$ pylori infection but not duodenal ulcer disease. The presence of $H$ pylori infection was confirmed by the ${ }^{14} \mathrm{C}$ urea breath test.

\section{SAMPLES}

Fasting gastric juice samples were aspirated into sterile universal containers containing $1 \mathrm{M}$ $\mathrm{HCl}$ and placed directly into a freezer at $-20^{\circ} \mathrm{C}$. Samples were transported for analysis frozen with dry ice and kept frozen at $-20^{\circ} \mathrm{C}$ until analysis.

\section{MEASUREMENT OF CYSTEAMINE}

Gastric juice specimens were prepared for high performance liquid chromatography (HPLC) following the amino acid chromatography method developed by Turnell and Cooper. ${ }^{14}$ Once samples were prepared, they were immediately injected onto a $7.5 \mathrm{~cm} 3 \mu \mathrm{m}$ spherisorb ODS column (HPLC Technology, Macclesfield, UK).

Elution of amino acid peaks was by gradient chromatography using a Shimadzu LC10 AD pump with a Shimadzu RF 535 fluorescence detector (Dyson instruments, Tyne and Wear, UK) set at $230 \mathrm{~nm}$ for excitation and $420 \mathrm{~nm}$ for emission. The gradient system consisted of solution A (water:propionic acid solution: acetonitrile, ratio $36: 10: 4$ ) and solution $B$ (water:acetonitrile:methanol:DMSO, ratio $21: 10: 17.5: 1.5) .{ }^{14}$ The gradient ran from $100 \%$ solution A to $100 \%$ solution B in five minute intervals over a total of 30 minutes. 


\section{Results}

Cysteamine was not found in any of the gastric juice samples or in a lysate of a laboratory culture of $H$ pylori. Colonies of the microorganism were extracted into $200 \mu \mathrm{l}$ of concentrated hydrochloric acid on four separate occasions. The detection limit of cysteamine by this method was $10 \mu \mathrm{mol} / 1$ and the peak eluted between 11.1 and 11.5 minutes. Each sample was run in duplicate with cysteamine $(125 \mu \mathrm{mol} / \mathrm{L})$ added to the second sample to confirm elution of cysteamine at around 11.1 minutes.

\section{Discussion}

This study has demonstrated that infection of the gastric mucosa by $H$ pylori does not result in the production of significant amounts of cysteamine in the gastric juice, indicating that this is not the mechanism through which $H$ pylori infection causes duodenal ulcer disease. $^{7-13}$

We chose to investigate cysteamine concentrations in fasting gastric juice samples because we considered that any small amounts of cysteamine present would be easily detectable in the relatively concentrated fasting gastric juice. Gastric juice also has a lower protein content than serum, which would reduce interference in the method from protein. Fasting samples were used because protein or sulphur containing amino acids in the diet could also have confounded attempts to measure small quantities of cysteamine.

It seems unlikely that concentrations of cysteamine below the detection limit of the assay would have been sufficient to cause duodenal ulcer disease, or that small amounts of cysteamine produced by $H$ pylori were missed. To induce duodenal ulcers in animal studies cysteamine was administered to rats at a dosage of $300 \mathrm{mg} / \mathrm{kg}$. This was sufficient to cause the depletion of immunoreactive somatostatin in the gastric mucosal cells within four hours of administration. ${ }^{78}$ An equivalent dose of cysteamine for a $70 \mathrm{~kg}$ man would be $21 \mathrm{~g}$, which if distributed equally, produces a theoretical average concentration of $6.5 \mathrm{mmol} / 1$ of cysteamine. The exact distribution of cysteamine following subcutaneous injection in rats is not known but as it is a small uncharged molecule it is likely to be widely and easily distributed through body fluids. As millimolar concentrations of cysteamine were required experimentally to produce ulcers, it seems likely that if cysteamine were produced by $H$ pylor $i$ the gastric juice concentrations of this compound should be of the same order of magnitude if it were a factor in the development of duodenal ulcer disease. In addition, cysteamine was not detected in supernatants of cultured $H$ pylori.

The depletion of gastric mucosal somatostatin is thought to be an important mechanism by which $H$ pylori infection leads to duodenal ulcer disease, however, it cannot be attributed to the effects of bacterial cysteamine production. Other causes of the depletion of somatostatin caused by $H$ pylori need to be considered. Possibilities include the effect of $H$ pylori ammonia production on antral surface $\mathrm{pH}^{15}$ or effects of cytokines produced by the mucosa in response to the bacterial infection.

We thank the North West Region of the Association of Clinica Biochemists for kindly supporting this project.

1 Langenberg ML, Tytgat GNJ, Schipper ME, Rietra PJG, Zanen HC. Campylobacter like organisms in the stomach of patients and healthy individuals [letter]. Lancet 1984;i: 1348

2 Neithercut WD, Williams C, Hossack MS, McColl KEL. Ammonium metabolism and protection from urease mediated destruction in Helicobacter pylori infection. $7 \mathrm{Clin}$ Pathol 1993;46:75-8.

3 Ferrero RL, Hazell SL, Lee A. The urease enzymes of Campylobacter pylori and a related bacterium. $f \mathrm{Med}$ Microbiol 1988;26:831-6.

4 Martensson J, Svensson H, Tobiasson P. Influence of the Martensson J, Svensson $H$, Tobiasson P. Influence of the
bacterial flora of the gut on sulphur amino acid degredation. Scand $\mathcal{f}$ Gastroenterol 1985;20:959-65.

5 Smith DA. S-aminoacid metabolism and its regulation in Escherichia coli and Salmonella typhimurium. Adv Genet 1971;16:141-65.

6 Soda K. Microbial sulphur amino acids an overview. Meth ods Enzymol 1987;143:453-9.

7 Ohe K, Miura Y, Taoka Y, Okada Y, Miyoshi A. Cysteamine induced inhibition of mucosal and pancreatic alkaline secretion in rat duodenium. Dig Dis Sci 1988;33:330-7.

8 Kapuscinski M, Shulkes A, Green M, Read D, MacLellan DG. Cysteamine can induce duodenal ulceration in rats DG. Cysteamine can induce duodenal ulceration in rats
without depleton of immunoreactive somatostatin. Regul Pept 1991;36:391-406.

9 Szabo S, Reichlin S. Somatostatin depletion by cysteamine: mechanism and implication for duodenal ulceration. Federation Proceedings 1985;44:2540-5.

10 Moss SF, Logan S, Bishop AE, Polak JM, Colain J. Effect of Helicobacter pylori on gastric somatostatin in duodenal ulcer disease. Lancet 1992;340:930-2.

11 Kantirek JW, Kantiret SJ, Domschke W. Cholecystokinin in the control of gastric secretion and gastric release in response to a meal at low and high pH in healthy subjects and duodenal ulcer patients. Scand $\mathcal{f}$ Gastroenterol 1995;30: and duoder

12 Tarnosky PR, Kovacs TOG, Sytrik B, Walsh JH. Asymptomatic Helicobacter pylori infection impairs $\mathrm{pH}$ inhibition of gastrin and acid secretion during second hour of peptone meal stimulation. Dig Dis Sci 1993;38:1681-7.

$13 \mathrm{McColl}$ KEL. Helicobacter pylori infection and abnormalities of acid secretion in patients with duodenal ulcer disease. Gastroenterology 1995;109:681-91.

14 Turnell DC, Cooper JDH. Rapid assay for amino acids in serum or urine by pre-column derivatization and reversed serum or urine by pre-column derivatization and reversed

15 Levi S, Beardshall K, Haddad G, Playford R, Ghosh P, Calam J. Campylobacter pylori and duodenal ulcers. The gastric link. Lancet 1989;i:1167-8. 